\title{
Delay-Throughput Performance in Mobile Ad-Hoc Networks with Heterogeneous Nodes
}

\author{
Valentina Martina * Michele Garetto ${ }^{\dagger} \quad$ Emilio Leonardi * \\ † Dipartimento di Informatica, Università di Torino, Italy \\ * Dipartimento di Elettronica, Politecnico di Torino, Italy
}

\begin{abstract}
In this paper, we analyze asymptotic delay-throughput performance of mobile ad-hoc networks comprising heterogeneous nodes with restricted mobility. In particular, we consider a scenario in which each node moves around one or more home-points (in a finite number) randomly placed over the area. For such restricted mobility model, we propose a new class of scheduling and routing schemes, which significantly outperforms all delay-throughput results previously obtained.
\end{abstract}

\section{INTRODUCTION}

Fundamental scaling laws ${ }^{1}$ of ad-hoc wireless networks have been extensively studied in the past several years, starting from the seminal works of Gupta-Kumar [1] and Grossglauser-Tse [2]. In the usual setting, $\Theta(n)$ nodes are placed over a finite region of the plane and randomly establish connections among them forming $\Theta(n)$ source-destination (S-D) pairs. Sources can employ multihop communications to reach their destinations, using other nodes as relays. Concurrent transmissions on the same wireless channel are subject to mutual interference, which limits the overall system performance.

In the case of mobile nodes, the novel store-carry-forward communication scheme, according to which nodes can physically carry data stored in their buffer before forwarding them to the next hop, has recently attracted lots of attention due to the increasing popularity of so called delay-tolerant networks [3] based on human [4] or vehicular [5] mobility. In this scenario, asymptotic analysis of capacity and delay as the number of nodes increases have being mainly carried out under the assumption that nodes are identical and uniformly visit the entire network area. Several mobility models have been analyzed, ranging from the simple re-shuffling model $[6,7]$, to the Brownian motion [8], and variants of random

$9{ }^{1}$ Given two functions $f(n) \geq 0$ and $g(n) \geq 0: f(n)=o(g(n))$ means $\lim _{n \rightarrow \infty} f(n) / g(n)=0 ; f(n)=O(g(n))$ means $\limsup _{n \rightarrow \infty} f(n) / g(n)=c<\infty ; f(n)=\omega(g(n))$ is equivalent to $g(n)=o(f(n)) ; f(n)=\Omega(g(n))$ is equivalent to $g(n)=$ $O(f(n)) ; f(n)=\Theta(g(n))$ means $f(n)=O(g(n))$ and $g(n)=$ $O(f(n))$; at last $f(n) \asymp g(n)$ means $\lim _{n \rightarrow \infty} f(n) / g(n)=1$. walk and random way-point $[9,10]$. All of the above mobility models are rather generous, since they allow the trajectory of each node to 'fill the space' over time, uniformly covering the entire network area. Such 'homogeneous mixing' assumption on the nodes' mobility process has been shown to provide optimal performance in terms of network capacity alone [2]. Whether this same assumption is also optimal in terms of delay-throughput trade-offs is an interesting question that we address in this paper, showing that uniform mobility can be largely suboptimal when both capacity and delay are considered.

Indeed, both every-day life experience and measurement data collected from real mobility traces $[11,12]$ suggest that the mobility pattern of an individual node is rather restricted over the network area, as users spend most of the time in proximity of a few preferred places, and rarely go outside their region of habit.

To the best of our knowledge, only few works have analyzed the impact of restricted mobility models on asymptotic throughput and delay. In [16] the authors consider a one-dimensional mobility model in which each node uniformly visits a randomly chosen great circle on the unit sphere, and obtain that a constant per-node throughput is achievable by each node, just like in the original Grossglauser-Tse scenario [2]. The work in [17] considers the same restricted mobility model, showing that also the delay is the same as in the case of uniform mobility over the entire network area.

In $[18,19]$ we have considered a two-dimensional, restricted mobility model which produces, for each node, a rotationally invariant stationary distribution centered at a home-point uniformly chosen in the area; the resulting throughput varies with continuity in between the two extreme cases of static nodes (Gupta-Kumar) and fully mobile nodes (Grossglauser-Tse), depending on how the physical network extension scales with respect to the average distance reached by the nodes from their home-point. This result confirms that throughput alone is maximized when the nodes span the entire network area.

In this work we use the same restricted mobility model introduced in $[18,19]$, extending the analysis to delay-throughput performance. To this aim we will adopt the simple re-shuffling model (also referred to as i.i.d. mobility model) already considered in [6,7] in the case of nodes uniformly visiting the network space. Our main finding is that restricted mobility can be very beneficial in terms of throughput and delay, as it allows to achieve superior performance (in scaling order) than in the case of homogeneous node mixing. This result suggests that realistic delay-tolerant networks, which are often characterized by restricted mobility patterns, can scale 
much better that what previously believed.

\section{SYSTEM ASSUMPTIONS}

\subsection{Mobility model}

We consider an extended network composed of $n$ nodes moving over a square region $\mathcal{O}$ of area $n$ with wrap-around conditions (i.e., a torus), to avoid border effects. Note that, under this assumption, the node density over the area remains constant and equal to 1 as we increase $n$. Time is divided into slots of equal duration, which is normalized to 1 .

In our work, we account for the restricted mobility pattern of nodes over the network area as follows. We assume that each node moves around one or more 'home-points' (in a finite number) randomly placed over the area. As an example, one home-point could be the residence of a person, and another home-point could be his/her workplace. At any given time we say that a node is associated to one of its home-points, meaning that it is currently moving in proximity of it. The node periodically migrates from one of its home-point to another. The exact details about how these migrations occur are not important. We simply assume that a node, while being associated to any of its home-points, spends on average a finite number of slots before migrating to another home-point. We further assume that the time taken to switch from one home-point to another is negligible (or at least uniformly bounded), and that a node does not have the opportunity of exchanging data with other nodes while traveling from one home-point to another (the contribution of such data exchanges to the asymptotic network capacity is, in any case, not important in order sense).

As a consequence of the above assumptions, each node spends a finite fraction of time in proximity of each of its home-points, and migrates in finite time from one home-point to another. In order to compute the resulting scaling laws of throughput and delay, such mobility process can be equivalently described by a twodimensional i.i.d. mobility model, in which the positions of the nodes are totally reshuffled after each slot, independently from slot to slot and among the nodes. At the beginning of each slot, a node jumps in zero time to a new position randomly selected according to a given spatial stationary distribution over the network area.

In our model, the overall spatial stationary distribution of a node can be obtained as follows. We first define the spatial stationary distribution of a node conditioned to the fact that it is currently associated to a given home-point. The overall spatial distribution is then given by a mixture of the spatial distributions of the node around its home-points, each of them weighted by the (positive) probability that the node is associated to the corresponding homepoint. It follows that we only need to specify: i) the stationary distribution of a node around each home-point; ii) the distribution over the network area of the home-points of a node.

Spatial distribution around each home-point. The spatial stationary distribution of a node around each of its home-points is assumed to be rotationally invariant with respect to the homepoint, and thus can be described by a generic, non increasing function $\phi(d)$ of the distance ${ }^{2} d$ from the home-point. In this paper we assume that $\phi(d)$ decays as a power-law of exponent $\delta$, i.e.,

$9^{2}$ Given any two points $X_{1}=\left(x_{1}, y_{1}\right) \in \mathcal{O} \quad$ and $X_{2}=\left(x_{2}, y_{2}\right) \in \mathcal{O}$ we define their distance over the torus surface as:

$\left\|X_{1}-X_{2}\right\|=\min _{u, v \in\{ \pm \sqrt{n}, 0\}} \sqrt{\left(x_{1}+u-x_{2}\right)^{2}+\left(y_{1}+v-y_{2}\right)^{2}}$ $\phi(d) \sim d^{-\delta}$. This choice is supported by a number of measurements papers which have found power-laws to be quite ubiquitous in experimental traces related to both human and vehicular mobility [12-15].

To avoid divergence problems in proximity of the home-point, we take function $s(d)=\min \left(1, d^{-\delta}\right)$, and normalize it so as to obtain a proper probability density function over the network area:

$$
\phi(d)=\frac{s(d)}{\iint_{\mathcal{O}} s(d)}
$$

The value of the normalization constant $G(\delta)=\iint_{\mathcal{O}} s(d)$ can be approximated, in order sense, by the following integral in polar coordinates:

$$
G(\delta)=\Theta\left(\int_{0}^{2 \pi} \mathrm{d} \theta \int_{0}^{\sqrt{n}} \min \left(1, \rho^{-\delta}\right) \rho \mathrm{d} \rho\right)
$$

We obtain that $G(\delta)$ is finite for any $\delta>2$. For $0 \leq \delta<2$ we have $G(\delta)=\Theta\left(n^{\frac{2-\delta}{2}}\right)$. For the special value $\delta=2$ we have $G(\delta)=\Theta(\log n)$. Note that $\delta=0$ leads to a uniform distribution over the space, whereas letting $\delta$ go to infinity we obtain the same behavior as that of a static network.

Spatial distribution of the home-points of a node. The spatial distribution of the home-points of a node is modelled as follows. One home-point, which is called the primary home-point of the node, is placed uniformly at random in the network area. Then, in the case of multiple home-points, all of the other home-points are randomly placed according to a rotationally invariant distribution centered at the primary home-point, which can be completely specified by a function $\psi(d)$ analogous to the one introduced above to describe the stationary distribution of a node around one homepoint, i.e., analogous to function $\phi(d)$ previously defined. Again, we assume that $\psi(d)$ decays as a power-law, with a different exponent $\zeta$, i.e., $\psi(d) \sim d^{-\zeta}$, with $\zeta<\delta$. We assume $\zeta<\delta$ because we want the home-points of a node to be well distinct from each other, and thus separated by an average distance larger than the typical distance reached by a node from one home-point. Otherwise, when $\zeta \geq \delta$, the network performance would be the same as in the case of a single home-point per node.

Since the number of home-points of a node is assumed to be uniformly bounded by a constant, it happens that all the performance gain (in terms of network capacity and delay) resulting from having multiple home-points per node can be already achieved in the case of just two home-points per node. For this reason, in the following we will restrict our analysis to the case in which each node $i$ has either a single home-point $H_{i}^{1}$, or two home-points $\mathbf{H}_{i}=\left\{H_{i}^{1}, H_{i}^{2}\right\}$.

Let $X_{i}(t)$ denote the position of node $i$ at time $t$ ( $t$ is an integer denoting the slot sequence number) and $\mathbf{X}(t)=$ $\left(X_{1}(t), X_{2}(t) \ldots X_{n}(t)\right)$ be the vector of nodes' positions at time $t$; we define by $d_{i j}(t)$ the distance between mobile $i$ and mobile $j$ at time $t$, i.e., $d_{i j}(t)=\left\|X_{i}(t)-X_{j}(t)\right\|$

We define by $d_{i j}^{H}$ the minimum distance between the home-points of nodes $i$ and $j$. In the case of a single home-point per node, $d_{i j}^{H}=\left\|H_{i}-H_{j}\right\|$. In the case of two home-points per node, we have

$$
d_{i j}^{H}=\min _{h, h^{\prime}=1,2}\left\{\left\|H_{i}^{h}-H_{j}^{h^{\prime}}\right\|\right\}
$$


One final assumption concerns the time-scale of node mobility with respect to the packet transmission time. In previous work dealing with asymptotic delay-throughput trade-offs, two different timescales have been analyzed: i) the fast-mobility case, in which only single-hop transmissions are allowed during a slot, being the packet transmission time comparable to the duration of a slot; ii) the slowmobility case, in which multi-hop communications can occur during a slot, by properly scaling down the packet transmission time. In this work we will focus on the more restrictive fast-mobility case, in which the packet transmission time matches the duration of a slot.

\subsection{Communication model}

To account for interference among simultaneous transmissions, we adopt the protocol model introduced in $[1]^{3}$. Nodes employ a common range $R$ for all transmissions which occur in the same time slot. A transmission from node $i$ to node $j$ using transmission range $R$ can be successfully received at node $j$ if and only if the following two conditions hold:

1. the distance between $i$ and $j$ is smaller than or equal to $R$, i.e., $d_{i j}(t) \leq R$

2. for every other node $k$ simultaneously transmitting, $d_{k j}(t) \geq(1+\Delta) R$, being $\Delta$ a guard factor.

Transmissions occur at fixed rate which is normalized to 1 . We assume that a single copy of each packet is present in the network at any time, i.e., data units are not broadcasted nor replicated, and nodes do not keep copies of previously received packets in their buffer.

\subsection{Traffic model}

Similarly to previous work we consider permutation traffic patterns in which $n$ randomly selected source-destination pairs exchange traffic at rate $\lambda$. Source-destination pairs are selected is such a way that every node is origin and destination of a single traffic flow with average rate $\lambda$. We further assume that messages are generated at every source according to independent memoryless Bernoullian processes.

\subsection{Throughput and delay}

We use the following definitions of asymptotic throughput and delay. Let $L_{i}(T)$ be the number of packets delivered to the destination of node $i$ in the time interval $[0, T]$. The delay of a packet is the time it takes to the packet to reach the destination after it is generated at the source. Let $D_{i}(t)$ be the sum of the delays experienced by all packets successfully delivered to the destination of node $i$ in the time interval $[0, T]$. We say that an asymptotic throughput $\lambda$ and an asymptotic delay $D$ per S-D pair are feasible if there is an $n_{0}$ such that for any $n \geq n_{0}$ there exists a scheduling/routing scheme for which both the following properties hold

$$
\begin{aligned}
& \lim _{T \rightarrow \infty} \operatorname{Pr}\left(\frac{L_{i}[T]}{T} \geq \lambda, \forall i\right)=1 \\
& \lim _{T \rightarrow \infty} \operatorname{Pr}\left(\frac{D_{i}[T]}{L_{i}[T]} \leq D, \forall i\right)=1
\end{aligned}
$$

$9^{3}$ The protocol model has been proven to be pessimistic with respect to the physical model employing power control (see Theorem 4.1, pag. 174 in [20]). Thus the results obtained in this paper can be regarded as lower bounds of the network performance achievable under the physical model employing power control.
Equivalently, we say in this case that the network sustains an aggregate throughput $\Lambda=n \lambda$. We will also adopt the simple Power Function [21], defined as the ratio $\lambda / D$, to characterize the system performance by a single metric.

\section{PREVIOUS RESULTS FOR THE I.I.D. FAST MOBILITY MODEL}

To avoid confusion, we limit ourselves to reporting existing scaling laws obtained for the i.i.d. mobility model, in the case of fast mobiles.

In [6] the authors analyze throughput/delay trade-offs in the case of nodes uniformly visiting the entire network area, with or without packet replication. The following general trade-off is established:

$$
\lambda=O\left(\frac{D}{n}\right)
$$

In particular the two-hop scheme of Grossglauser-Tse is proven to incur a delay $D=\Theta(n)$ while guaranteeing a per-node throughput $\lambda=\Theta(1)$. To improve delay, two different schemes exploiting packet redundancy are proposed: the first is still based on two hops, and achieves $D=\Theta(\sqrt{n})$ and $\lambda=\Theta(1 / \sqrt{n})$; the second employs multiple hops and achieves better delay performance $D=O(\log n)$ while sacrificing the per-node throughput $\lambda=O(1 /(n \log n))$.

It has been shown in [7] that previous results can be further improved by encoding transmitted information at sources. A class of joint coding-scheduling-routing schemes is introduced which achieves a trade-off

$$
\lambda=\Theta\left(\sqrt{\frac{D}{n}}\right)
$$

in the case of i.i.d. mobility with fast mobiles, when $D$ is both $\omega(\sqrt[3]{n})$ and $o(n)$.

In [22] we have already analyzed the delay-throughput trade-offs achievable under the restricted mobility model considered here, limiting our attention to the case of a single home-point per node. However, in [22] we have proposed a class of scheduling-routing schemes specifically tailored to the case of $\delta<2$, i.e., for nodes with broad mobility over the network area. Such schemes turns out to be largely suboptimal when $\delta>2$, i.e., for more restricted mobility patterns. With respect to [22], in this paper we provide the following contributions: i) we propose a different class of scheduling-routing schemes, which are tailored to the case of $\delta>2$, and achieve much better performance than that reported in [22]; ii) we were able to prove that our scheme jointly achieves near-optimal throughput and delay performance for $2 \leq \delta<3$; iii) we analyze the case of multiple home-points per node, showing how the network performance can be improved by exploiting restricted mobility patterns around different home-points.

\section{ALGORITHM DESCRIPTION 4.1 The routing scheme}

We propose a routing scheme according to which messages advance along a chain of relay nodes whose home-points become progressively closer (in terms of the minimal home-point distance specified by (1)) to the home-points of the destination.

More in detail, we recursively partition the network area into squarelets of smaller and smaller size. At the first iteration the do- 


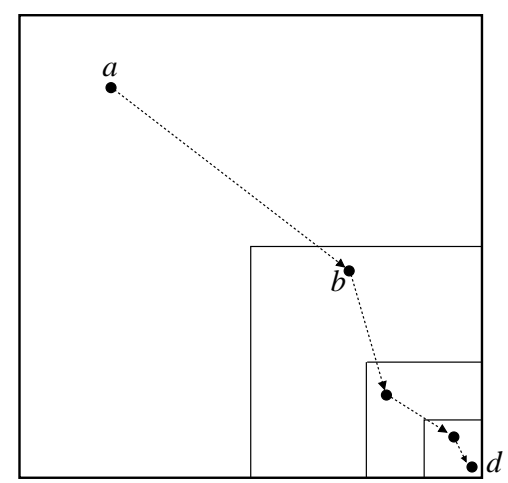

Figure 1: Example of routing of a message generated by node $a$ in routing step 0 . Dots denote home-points of nodes.

main $\mathcal{O}$ is divided into four disjoint squares of edge $\sqrt{n} / 2$. At each iteration $i$ ( $\left.i \leq i_{\max }\right)$ every square obtained in the previous iteration is in turn divided into four disjoint squarelets. By so doing, $i$-level squarelets have edge $z_{i}=\sqrt{n} / 2^{i}$, and their cardinality is $4^{i}$. We denote by $\mathcal{S}_{k}^{i}$, for $1 \leq k \leq 4^{i}$, each $i$-level squarelet, $0 \leq i \leq i_{\max }$. We say that two $i$-level squares are adjacent if they are originated from the same $(i-1)$-level square. Notice that $S_{1}^{0}=\mathcal{O}$. Parameter $i_{\max }$ determines the edge of the smallest squarelet, $z_{i_{\max }}=\sqrt{n} / 2^{i_{\max }}$. At last, we denote by $\left|\mathcal{S}^{i}\right|=z_{i}^{2}$ the area of a level- $i$ squarelet.

We say that two nodes $a$ and $b$ are $i$-level neighbors if the smallest squarelet containing both a home-point of $a$ and a home-point of $b$ is a $i$-level squarelet. Suppose that node $a$ has to forward a packet $m$ directed to a destination $d$ which is a $i$-level neighbor of $a$. In this case, while being handled by $a$, we say that $m$ is in step $i$ of the routing algorithm.

If $i<i_{\max }$, node $a$ sends the packet to a node $b$ which is an $(i+1)$ level neighbor of $d$. If $i=i_{\max }$, node $a$ sends the packet directly to its final destination $d$. By construction, packets go through at most $i_{\max }$ intermediate relay nodes before being delivered to the destination. Figure 1 illustrates an example in which $i_{\max }=3$, and a message is generated by node $a$ in routing step 0 , destined to node $d$. Here the message has to go through 3 relay nodes before arriving in a squarelet of minimum size containing the home-point of the destination.

To guarantee the existence, uniformly in the network, of nodes $b$ which are $(i+1)$-level neighbors of $d$, we need to have $\left|\mathcal{S}^{i}\right|=$ $\Omega(\log n)$ for any $i \leq i_{\max }$. This comes from a standard concentration result of the Poisson point process widely used in related work, and not repeated here. As a consequence, the smallest possible edge is $z_{i_{\max }}=\Omega(\sqrt{\log n})$, where $i_{\max }=1 / 2 \log _{2} n-$ $\log _{2} z_{i_{\max }} \leq 2 \log _{2} n-\log _{2} \log n=\Theta(\log n)$. In the following we will better specify how to properly select $z_{i_{\max }}$.

\subsection{The scheduling scheme}

A scheduling scheme is in charge of selecting, at each slot, the set of (non-interfering) transmitter-receiver pairs to be enabled in the network, as well as the message to be transmitted over each enabled pair. In our family of schemes, the transmission range employed by a transmitter depends on the routing step reached by the message to be sent. To better pack simultaneous transmissions, and thus maximize the network throughput, our scheduling policy selects,

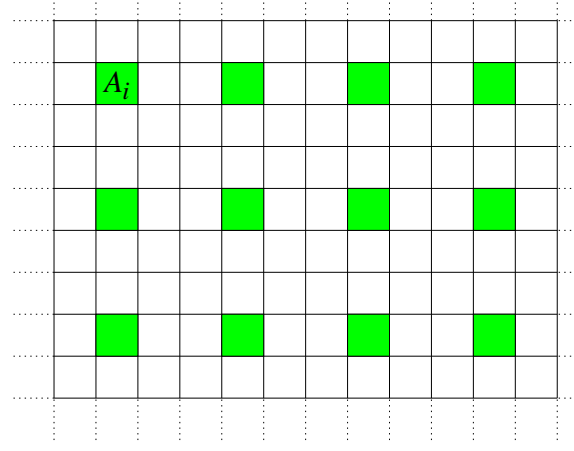

Figure 2: Example of non-interfering subset of cells with $M=$ 9.

in a given slot, transmissions having homogeneous transmission ranges. This is done by selecting, at the beginning of each slot, a step $i$ according to an assigned probability distribution $p_{i}^{s}$. Then the slot is reserved only to messages which are in step $i$ of the routing algorithm, i.e., to messages currently stored at nodes which are $i$ level neighbors of the destination.

A common transmission range $R_{i}=O\left(\frac{\sqrt{n}}{2^{i}}\right)$ is employed by all communications occurring during a slot devoted to step $i$ of the routing scheme. Once step $i$ has been selected, every squarelet $\mathcal{S}_{k}^{i}$ is divided into square cells $\mathcal{A}_{i}$ of area $A_{i}=R_{i}^{2}=O\left(z_{i}^{2}\right)$, forming a regular square tessellation.

According to the protocol model, at most one transmission can be enabled in each cell. Moreover, one can easily construct $M=$ $\Theta(1)$ subsets of regularly spaced, non-interfering cells (for example, $M=9$ assuming a protocol model with $\Delta=0$, see Figure 2). Each subset can then be enabled in one out of $M$ slots, guaranteeing fairness among all cells and absence of interference among concurrent transmissions.

Within a given cell $\mathcal{A}_{i}$, the specific transmitter-receiver pair to be enabled is selected as follows. First a node $a$ currently staying in $\mathcal{A}_{i}$ and such that: i) $a$ has a home-point in $\mathcal{A}_{i}$; ii) $a$ is storing a message $m$ in routing step $i$, is selected at random as transmitter, if there is any. Let $d$ be the final destination of message $m$. If $i=i_{\max }$, node $d$ is the only possible receiver, hence it must be found in $\mathcal{A}_{i}$ for the communication to occur. Instead, for $i<i_{\max }$, a node $b$ (if there is any) is randomly selected as receiver among the $(i+1)$-level neighbors of $d$ currently staying in $\mathcal{A}_{i}$. Note that by construction home-points of $a$ and $b$ lie in adjacent $(i+1)$-level squares.

If such a transmitter-receiver pair is found, cell $\mathcal{A}_{i}$ is declared as active and the transmission from $a$ to $b$ ( $d$ in the case of $i=i_{\max }$ ) takes place.

We further assume that messages $m$ in routing step $i \leq i_{\max }$ are selected by node $a$ according to a FIFO scheduling policy. Thus, logically each node is equipped with a FIFO queue for each routing step (Figure 3).

\section{PERFORMANCE EVALUATION}

\subsection{Design considerations}


Given that step $i$ has been selected at the beginning of a slot, by construction the number $N_{i}$ of parallel transmissions that can occur in the network during the slot equals the number of active cells. On average we have:

$$
\mathbb{E}\left[N_{i}\right]=\frac{n}{M A_{i}} \mathbb{P}(\text { active cell } \mid i)
$$

where $n$ is the total network area, $M=\Theta(1)$ is a constant accounting for interference, and $\mathbb{P}($ active cell $\mid i)$ is the probability that a generic cell is active at step $i$.

Note that, on the one hand, areas $A_{i}$ should be minimized so as to maximize the spatial reuse; on the other hand, if areas $A_{i}$ are set too small then $\mathbb{P}$ (active cell $\mid i$ ) becomes arbitrarily small as well, vanishing the potential advantage of increasing the spatial reuse. A good design choice is to minimize $A_{i}$ while guaranteeing that $\lim _{n \rightarrow \infty} \mathbb{P}($ active cell $\mid i)>0$.

Unfortunately a direct evaluation of $\mathbb{P}($ active cell $\mid i$ ) is rather difficult, because $\mathbb{P}$ (active cell $\mid i$ ) depends on both node positions and traffic conditions. Thus we upper-bound $\mathbb{P}($ active cell $\mid i)$ with $\mathbb{P}$ (populated cell $\mid i$ ), which is the probability that cell $\mathcal{A}_{i}$ is populated by at least one pair of nodes $(t, r)$ in which node $t$ is a potential transmitter for a message $m$ in step $i$, and $r$ is a potential receiver of message $m$.

Note that the occurrence of the event $\left\{\right.$ cell $\mathcal{A}_{i}$ is populated $\}$ is necessary for the occurrence of the event $\left\{\right.$ cell $\mathcal{A}_{i}$ is active $\}$. Furthermore observe that $\mathbb{P}$ (populated cell $\mid i$ ) coincides with $\mathbb{P}($ active cell $\mid i)$ in saturated traffic conditions, i.e., when nodes are constantly backlogged with step- $i$ messages to transmit.

The event $\left\{\right.$ cell $\mathcal{A}_{i}$ is populated $\}$ no longer depends on traffic conditions, but only on the positions of the nodes in the current step. This fact allows us to design areas $A_{i}$ using only geometric considerations. In particular, when $i=i_{\max }, t$ is required to have a home-point in $\mathcal{A}_{i}$, whereas $r$ is the final destination of message $m$, thus it must have a home-point in the same $i_{\max }$-level squarelet containing $\mathcal{A}_{i}$. For $i<i_{\max }, t$ is required to have a home-point in $\mathcal{A}_{i}$, whereas $r$ must have a home point in the $(i+1)$-level squarelet containing the destination.

As we will see, by an appropriate choice of $A_{i}$, it is possible to guarantee that $\lim _{n \rightarrow \infty} \mathbb{P}$ (populated cell $\mid i$ ) $>0$ irrespective of the specific message $m$ in step $i$ stored by $t$. Moreover we will show that, with our choice of $A_{i}$, the network queues can be loaded is such a way that $\mathbb{P}($ active cell $\mid i)=\Theta(\mathbb{P}($ populated cell $\mid i))$ i.e., the probability to find a transmitter $t$ holding a message $m$ in step $i$ is $\Theta(1)^{4}$. This will permit us to conclude that our design of areas $A_{i}$ is indeed optimal (in order sense) also considering queueing effects.

To compute the minimum value of $A_{i}$ which guarantees that $\lim _{n \rightarrow \infty} \mathbb{P}$ (populated cell $\mid i$ ) $>0$, we separately consider the number of candidate transmitters (nodes $t$ ) and the number of candidate receivers (nodes $r$ ) within $\mathcal{A}_{i}$.

\section{Lemma 1. At any given slot, the number of nodes thaving one}

$9^{4}$ This condition can be satisfied, more precisely, for the routing step that determines the overall network capacity, i.e., the system bottleneck. home-point in $\mathcal{A}_{i}$ and currently staying in $\mathcal{A}_{i}$ is $\Theta\left(A_{i}\right)$ w.h.p., provided that $A_{i}=\Omega(\log n)$.

Proof. see Appendix. A

Lemma 1 allows to say that, to guarantee the presence of at least one transmitter in $\mathcal{A}_{i}$, it is sufficient to have $A_{i}=\Omega(\log n)$. The above lemma is essentially a consequence of concentration results for the distribution of the home-points and of the fact that, when $\delta>2$, each node spends a finite fraction of time within a finite distance from its home-point.

We first consider separately the last step $i=i_{\max }$, in which $t$ transmits directly to the final destination of the message. Recall that, by construction, $\left|S^{i_{\max }}\right|=\Omega(\log n)$ : therefore, by setting $\mathcal{A}_{i_{\max }}=\left|S^{i_{\max }}\right|$, we can be sure that, for any chosen transmitter $t$, the destination can be found in $\mathcal{A}_{i_{\max }}$ with non-null probability irrespective of the specific message in step $i=i_{\max }$ stored by $t$.

We now consider the generic step $i<i_{\text {max }}$. By lemma 1 the presence of at least one transmitter $t$ in $\mathcal{A}_{i}$ is guaranteed by $A_{i}=$ $\Omega(\log n)$. For the generic message in step $i$ stored by $t$, there are essentially three classes of potential receivers $r$ that can be found in $\mathcal{A}_{i}$ :

1. nodes $b$ having one home-point placed in the same $(i+1)$ level squarelet of the destination, and currently staying in $\mathcal{A}_{i}$ for effect of mobility around such home-point, at distance $\Theta\left(z_{i}\right)$.

2. nodes $b$ having one home-point $H_{b}^{h}$ in the same $(i+1)$ level squarelet of the destination, another home-point $H_{b}^{3-h}$ placed in $\mathcal{A}_{i}$, and currently moving within $\mathcal{A}_{i}$ for effect of mobility around $\hat{H}$.

3. nodes $b$ having one home-point $H_{b}^{h}$ in the same $(i+1)$-level squarelet of the destination, another home-point $H_{b}^{3-h}$ not placed in $\mathcal{A}_{i}$, and currently moving within $\mathcal{A}_{i}$ for effect of mobility around $H_{b}^{3-h}$.

Notice that classes 2 and 3 require nodes to have multiple homepoint, otherwise in the case of a single home-points per node the only contribution comes from class 1 .

We denote by $\Phi_{i}^{j}, j=1,2,3$, the density of receivers within $\mathcal{A}_{i}$ belonging to the above three classes, and by $\Phi_{i}=\sum_{j=1}^{3} \Phi_{i}^{j}$ the overall density of receivers within $\mathcal{A}_{i}$. Then the optimal choice for $A_{i}$ is to set $A_{i}=1 / \Phi_{i}$, as proved in [23] (Lemma 2 ). Notice that the overall density of receivers in $\mathcal{A}_{i}$ is equivalent (in order sense) to the largest among the $\Phi_{i}^{j}$, s, hence $A_{i}=\Theta\left(\left(\max \left\{\Phi_{i}^{1}, \Phi_{i}^{2}, \Phi_{i}^{3}\right\}\right)^{-1}\right)$.

As shown in Appendix B, we have $\Phi_{i}^{1}=\Theta\left(z_{i}^{2-\delta}\right) ; \Phi_{i}^{2}=$ $\Theta\left(z_{i}^{2-\zeta} / G(\zeta)\right) ; \Phi_{i}^{3}=O\left(\max \left(\Phi_{1}, \Phi_{2}\right)\right)$. Neglecting the contribution of the third class, it remains to understand which one between the first two classes provides the higher density of candidate receivers. It turns out that this can depend not only on the values of the exponents $\delta$ and $\zeta$, but also on $z_{i}$, i.e. on the current step $i$.

We identify the following three cases: 
- Single home-point per node. In this case there is only one-class of receivers, whose density is $\Phi_{i}^{1}$, hence $A_{i}=$ $\Theta\left(z_{i}^{\delta-2}\right), \forall i$. We observe that $\Phi_{i}^{1}$ decreases with $z_{i}$, hence the crucial step (the one that requires the largest cells, of area $A_{i}$, in order to find candidate receivers) is the first one (corresponding to $i=0)$. To guarantee that $A_{i}=O\left(z_{i}^{2}\right)$ it must be $\delta \leq 4$. At last, observe that Lemma 1 requires $A_{i}$ to be $\Omega(\log n)$ for any $i$. Thus $z_{i_{\max }}$ must be $\Omega\left((\log n)^{\frac{1}{\delta-2}}\right)$. As a result, we select $z_{i_{\max }}=\Theta\left((\log n)^{\frac{1}{\delta-2}}\right)$. Notice that with this choice we also satisfy the condition $z_{i_{\max }}=$ $\Omega(\sqrt{\log n})$, for any $\delta \leq 4$.

- Multiple home-points per node, $\zeta>2$. In this case, recalling that we assume $\zeta<\delta$ so as to have well distinct home-points per node, we have $\Phi_{i}^{2}=\omega\left(\Phi_{i}^{1}\right)$, hence $A_{i}=\Theta\left(z_{i}^{\zeta-2}\right), \forall i$ (we remind that for $\zeta>2$ we have $G(\zeta)=\Theta(1)$ ). Again, the first step is the one requiring the largest value of $A_{i}$. To guarantee that $A_{i}=O\left(z_{i}^{2}\right)$ it must be $\zeta \leq 4$. Similarly to the previous case $z_{i_{\max }}$ must be $\Omega\left((\log n)^{\frac{1}{\zeta-2}}\right)$; thus we select $z_{i_{\max }}=\Theta\left((\log n)^{\frac{1}{\zeta-2}}\right)$.

- Multiple home-points per node, $\zeta<2$. This is the most interesting case, since here $\Phi_{i}^{1}$ decreases with $z_{i}$, whereas $\Phi_{i}^{2}=\Theta\left(z_{i}^{2-\zeta} / n^{(2-\zeta) / 2}\right)$ increases with $z_{i}$. The optimal strategy is to exploit receivers belonging to the second class for all of the initial steps characterized by $z_{i}>z_{i^{*}}$, where $z_{i^{*}}$ is a critical distance from the destination, and then to rely on receivers belonging to the first class to deliver the message up to the final destination. This means that the presence of multiple home-points is employed to advance the message up to the critical distance $z_{i^{*}}$, after which the mobility around a single home-point is used. The critical distance $z_{i}$ can be easily computed by looking for the step $i^{*}$ at which $\Phi_{i}^{1}=$ $\Theta\left(\Phi_{i}^{2}\right)$. It turns out that $z_{i^{*}}=\Theta\left(n^{\frac{2-\zeta}{2(\delta-\zeta)}}\right)$. Notice that in this case the largest cell size among all steps is $A_{i^{*}}=z_{i^{*}}^{\delta-2}$. At last, to guarantee that $A_{i}=O\left(z_{i}^{2}\right)$ it must be $\delta \leq 4$. In this case we select $z_{i_{\max }}=\Theta\left((\log n)^{\frac{1}{\delta-2}}\right)$.

Note that in all cases above we have $i_{\max }=\Theta(\log n)$. The cases in which $A_{i}=O\left(z_{i}^{2}\right)$ does not hold require to adopt alternative schemes that will be described later in Section 6.

Having found the optimal value of $A_{i}$, from (4) it turns out that the number of parallel transmissions $\mathbb{E}\left[N_{i}\right]$ is upper-bounded by $\overline{N_{i}}=\frac{n}{A_{i}}$.

We now turn our attention to the probability distribution $p_{i}^{s}$ according to which slots are assigned to the different steps of the routing algorithm. To maximize the system throughput a natural choice would be to equalize the average number of transmissions that can occur at each step, and thus avoid that a particular step becomes the system bottleneck; this is obtained by making $p_{i}^{s}$ inversely proportional to $\bar{N}_{i}$. This choice has been pursued in [22], where optimal delay-throughput trade-offs have been investigated for the case of $\delta<2$. However this approach, when applied to our class of scheduling-routing schemes specifically tailored to the case $\delta>2$, results into excessively large delays, because some steps turn out to be scheduled very rarely.

An alternative strategy, which allows to obtain significant better delays at the expense of just a logarithmic penalty in system through-

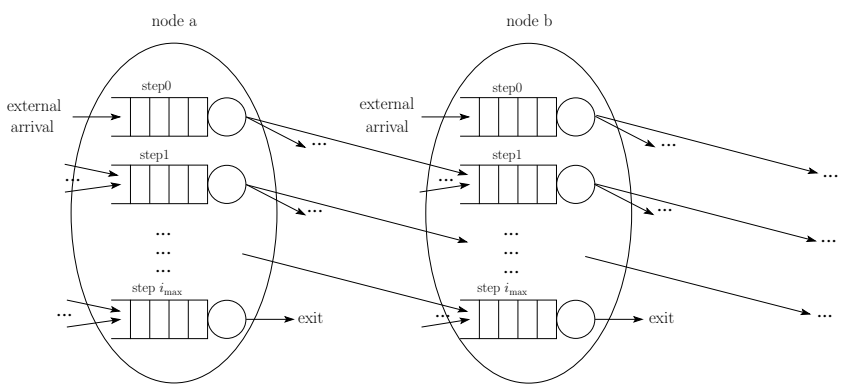

Figure 3: Queue structure of the system. Every node is equipped with one FIFO queue for each step.

put, is to assign an equal probability to each routing step:

$$
p_{i}^{s}=\frac{1}{i_{\max }+1}
$$

We have decided to adopt this slot assignment to obtain the best throughput-delay performance by our new class of schedulingrouting schemes. With all these design choices, an upper bound to the traffic that can be transferred through the network is provided by:

$$
\bar{\Lambda}=\min _{i} p_{i}^{s} \bar{N}_{i}=\left\{\begin{array}{cc}
\Theta\left(\frac{n^{1+\frac{2-\delta}{2}}}{\log n}\right) & \text { single home-point, } \\
\Theta\left(\frac{n^{1+\frac{2-\zeta}{2}}}{\log n}\right) & 2<\delta<4 \\
\Theta\left(\frac{n^{1+\frac{(2-\zeta)(2-\delta)}{2(\delta-\zeta)}}}{\log n}\right) & \text { multiple home-points, } \\
& 2<\zeta<4 \\
& \zeta<2,2<\delta<4
\end{array}\right.
$$

We recall that the above expression provides the system throughput in saturated conditions, i.e., when nodes are constantly backlogged by messages to send, at any step.

\subsection{Queueing dynamics; maximum through- put}

As illustrated in Figure 3, the system can be represented as an open network of queues, where queues correspond to the physical FIFO queues present in the nodes (recall that in any node there is one queue for each routing step).

The resulting queueing network is acyclic. Indeed, messages advance in the network visiting queues associated to increasing step indices $i$, which guarantees the absence of loops (see Figure 3).

Therefore, considering that traffic is split evenly (for symmetry among the queues associated to each routing step) among the queues storing messages in the same routing step, we can be sure that no queue is overloaded by limiting the offered load $\Lambda=n \lambda$ to a value strictly less than the saturated system throughput $\bar{\Lambda}$.

We conclude that offered load $\Lambda=O(\bar{\Lambda})$ can be successfully transferred through the network. In particular, the maximum achievable throughput according to our scheme is $\Lambda=\Theta(\bar{\Lambda})$, and in this case, at least for the index $i^{*}$ such that $p_{i^{*}}^{s} \bar{N}_{i^{*}}$ is the minimum throughput among all steps, we have $\mathbb{P}\left(\right.$ active cell $\left.\mid i^{*}\right)=$ $\Theta\left(\mathbb{P}\left(\right.\right.$ populated cell $\left.\mid i^{*}\right)$. 


\subsection{Delay analysis}

To analyze the delay performance, it is more convenient to look at the system from a different perspective, in which we associate one virtual queue to each cell, for all steps. A virtual queue stores all messages in a given routing step which have to be transmitted in one of the cells associated to the step. We obtain also in this case an open, acyclic queueing network, since messages visit virtual queues associated to increasing step indices.

We observe that, in a generic slot, a transmission in a given virtual queue associated to step $i$ takes place, provided that the considered queue is not empty, if the following conditions hold: i) the considered slot is devoted to step $i$; ii) the corresponding cell belongs to the subset of non interfering cells that are allowed to transmit; iii) the scheduling policy is able to identify within the corresponding cell a pair of nodes $(t, r)$, which can act respectively as transmitter and receiver of a message enqueued in the considered virtual queue. We model the considered virtual queue as a single server queue, of which we characterize the service time and the arrival process.

We start from the service time. Note that the probability of joint occurrence of conditions i) and ii) above is $p_{i}^{s} / M$, independently from slot to slot. Slightly more difficult is the evaluation of the occurrence probability of condition iii), since it depends on the number of messages stored in the considered virtual queue; indeed messages enqueued in the virtual queue can be transmitted only if the nodes physically carrying them are currently staying in the corresponding cell. The probability that at least one such transmitters is found within the corresponding cell increases as the number of messages stored in the virtual queue increases. However, we can clearly bound (from below) the occurrence probability of iii), assuming that just a single message is stored in the virtual queue at any time. In this latter case, the probability of finding a $(t, r)$ pair can be easily shown to be $\Theta(1)$ as result of our design choices. Indeed, considering a node $t$ having a packet to transmit in step $i$. By construction it has one home-point $H_{t}^{h}(h=1$ or 2$)$ within a cell $\mathcal{A}_{i}$ of area $A_{i}=\Omega(\log n)$. Thus, the probability that node $t$ can be found in $\mathcal{A}_{i}$ is $\mathbb{P}\left(t \in \mathcal{A}_{i} \mid H_{t}^{h} \in \mathcal{A}_{i}\right)=\Theta(1)$ as shown in Appendix A. Now, since the average density of potential receivers $r$ in $\mathcal{A}_{i}$ is $\Theta\left(1 / A_{i}\right)$, the average number of potential receivers within $\mathcal{A}_{i}$ is $\Theta(1)$. This implies that the probability of finding at least one receiver within $\mathcal{A}_{i}$ is $\Theta(1)$ as well.

Previous arguments permit to conclude that service times of the considered virtual queue can be dominated by a sequence of geometrically distributed i.i.d. service times with average $\Theta\left(1 / p_{i}^{s}\right)=$ $\Theta\left(i_{\max }\right)$.

The arrival process of packets to the virtual queue is, instead, given by the superposition of the arrival processes to the physical queues associated to step $i$ of the nodes having one home-point in the corresponding cell (the number of such nodes in $\Theta\left(A_{i}\right)$ ).

For the initial step $i=0$, it is just the superposition of the independent Bernoullian processes according to which packets are generated at nodes. The analysis of the following steps $i>0$ is, instead, slightly more complex. Each node $b$ having one home-point in the corresponding cell can potentially bring a packet to the virtual queue in a given slot, if in the considered slot it acts as receiver for a message in routing step $j=i-1$.

This can happen under the following conditions: i) node $b$ moves to a distance $\Theta\left(z_{i}\right)$ from its home-point; ii) the slot is assigned to step $j=i-1$; iii) the visited cell has a packet to transmit; iv) the node is chosen as receiver among the candidate receivers in the same cell belonging to step $j$.

The satisfaction of the above conditions (especially condition iii)) can not be easily evaluated. However, observe that, provided that the slot is assigned to step $j=i-1$, by construction the number of simultaneous arrivals at the considered virtual queue is upperbounded by the number of nodes having an home-point in $\mathcal{A}_{i}$ that are concurrently staying at a distance $\Theta\left(z_{i}\right)$ from $\mathcal{A}_{i}$.

The number of such nodes is i) binomially distributed since positions of different nodes are i.i.d. (it tends to a Poisson distribution in the large $n$ limit); ii) it regenerates from slot to slot. iii) its average is $\Theta(1)$ as shown in Appendix C.

As a result of previous arguments, although the dynamics of a virtual queue associated to step $i$ may exhibit complex dependencies over time, the number of messages in it can be stochastically dominated by the number of messages in a $G e o^{[X]} / G e o / 1$ (a $G e o / G e o / 1$ queue with bulk arrivals), in which the service times (batch sizes) are memoryless and lower (upper)-bounded as explained above.

Thus, the average delay $E\left[D^{i}\right]$ experienced by step- $i$ messages in the considered virtual queue can be bounded using the PollacekKinchine formula [24]:

$$
E\left[D^{i}\right] \leq s_{i}+\frac{a^{2} s_{i}^{2}-a\left(s_{i}^{(2)}+s_{i}\right)+a^{(2)} s_{i}^{2}}{2\left(1-a s_{i}\right)}
$$

where $s_{i}$ and $s_{i}^{(2)}$ are, respectively, the first and the second moment of the service time for the dominating $G e o^{[X]} / \mathrm{Geo} / 1$ queue, while $a$ and $a^{(2)}$ are, respectively, the first and the second moment of the batch size distribution (which is binomial both in the case $i=0$ and in the case $i>0$ ).

From the above considerations on service times and batch size distributions it follows that: $s_{i}=\Theta\left(i_{\max }\right), s_{i}^{(2)}=\Theta\left(i_{\max }^{2}\right)$, $a_{i}=\Theta\left(1 / i_{\max }\right)$ for every $i$, while $a_{i}^{(2)}$ is $\Theta\left(1 / i_{\text {max }}^{2}\right)$ for $i=0$ and $\Theta\left(1 / i_{\max }\right)$ for $i>0$.

In conclusion, recalling that $i_{\max }=\Theta(\log n)$, it turns out that $E\left[D^{i}\right]=\Theta(\log n)$ for every step $i$.

At last, since messages are forwarded to destinations through $O\left(i_{\max }\right)=O(\log n)$ steps, the overall delivery delay is $\mathbb{E}[D]=$ $O\left((\log n)^{2}\right)$.

\section{ALTERNATIVE SCHEMES LARGE VALUES OF $\delta$ AND $\zeta$}

FOR

We recall that our previously described scheduling-routing schemes require that $A_{i}=R_{i}^{2}=O\left(z_{i}^{2}\right)$. This condition does not hold when $\delta>4$, or when $\zeta>4$ (in the case of multiple home-points).

In the case of multiple home-points having $\zeta<2$ and $\delta>4$, it is still possible to apply our bisection scheme up to a minimum distance $z^{*}$ from the destination, and then resort to a direct transmission to reach the destination. Indeed, no matter how big is $\delta$, i.e., how much restrictive is the mobility of nodes around each home point, we can still exploit the presence of multiple home-point per node to efficiently advance the message in the beginning of the route, when $z_{i}>z^{*}$. The value of $z^{*}$ can be computed consid- 
ering that the density of receivers is at least $\Phi_{i}^{2}=\Theta\left(z_{i}^{2-\zeta} / G(\zeta)\right)$, and thus condition $A_{i}=R_{i}^{2}=O\left(z_{i}^{2}\right)$ provides $z^{*}=\Theta\left(n^{\frac{2-\zeta}{2(4-\zeta)}}\right)$. When the message has reached distance $z^{*}$ from the destination, it can be sent directly to the destination by a single hop which introduces a delay of $\Theta(1)$, since every node spends a constant fraction of time in proximity of its home-point. The above alternative schemes provides a throughput

$$
\Lambda=\Theta\left(\frac{n^{1-\frac{2-\zeta}{4-\zeta}}}{\log n}\right) \quad \text { multiple home-points, } \zeta<2, \delta>4
$$

and delay $D=O\left((\log n)^{2}\right)$. It can be observed that this is the same performance achieved by our original scheme when $\delta=4$.

In the case of a single-home point per node with $\delta>4$, or multiple home-point per node with $\zeta>4$, instead, our bisection scheme cannot be applied even in the first steps. In this cases it is necessary to resort to an alternative scheme valid for any value of $\delta>2$, that does not exploit neither the multiplicity of home-points nor the mobility of nodes.

This scheme is based on the observation that, for any $\delta>2$, every node spends a constant fraction of time within a finite distance from its home-point. Hence, by enabling the nodes to communicate only when they are in proximity of their home-points, we can apply the classic Gupta-Kumar scheme for fixed nodes at thus achieve at least the same performance as that of a static network.

Recall from $[1,8]$ that a static network with uniform node density sustains a per-node throughput $\lambda=\Theta(1 / \sqrt{n \log n})$, while incurring a delay $D=\Theta(\sqrt{n / \log n})$. More in general, it allows to achieve delay-throughput trade-offs characterized by $\lambda / D=$ $\Theta(1 / n)$, for all $\lambda=O(1 / \sqrt{n \log n})[8]$.

\section{SUMMARY OF RESULTS}

To summarize our results, we report the scaling exponent ${ }^{5}$ of the power function $\lambda / D$ achievable by our family of schedulingrouting schemes, in all considered cases:

$e(\lambda / D)=\left\{\begin{array}{l}\frac{2-\delta}{2} \\ \frac{2-\zeta}{2} \\ -1 \\ \frac{(2-\zeta)(2-\delta)}{2(\delta-\zeta)} \\ \frac{2-\zeta}{\zeta-4}\end{array}\right.$

single home-point, $\delta \leq 4$ multiple home-points, $2<\zeta \leq 4$

single home-point, $\delta>4$ or multiple home-points, $\zeta>4$

multiple home-points, $\zeta<2, \delta \leq 4$ multiple home-points, $\zeta<2, \delta>4$

Figure 4 offers a 3D representation of (8). In Figure 4, points characterized by $\zeta>\delta$ represent nodes having a single home-point.

In Figure 5 we report a 2D plot of the same results restricting our attention to cases in which $\zeta<2$, or in which nodes have a single home-point. In particular, the curve labelled ' 1 h.p.' corresponds to the novel scheduling-routing scheme proposed in this work for the single home-point case, whereas the curve labelled ' 1 h.p. old scheme' refers to the performance achievable in the same case by the scheme proposed in [22], characterized by $e(\lambda / D)=2-\delta$. Figure 5 shows that, when $\delta>2$, the scheme proposed in this work

$9^{5}$ The scaling exponent of a generic function $f(n)$ is defined as $e(f):=\lim _{n \rightarrow \infty} \frac{\log f(n)}{\log n}$.

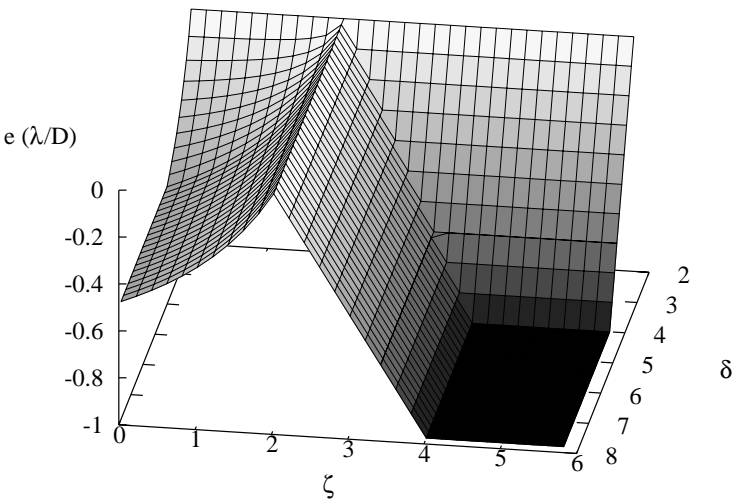

Figure 4: Scaling exponent of the power achievable by the proposed schemes for different values of $\zeta$ and $\delta$.

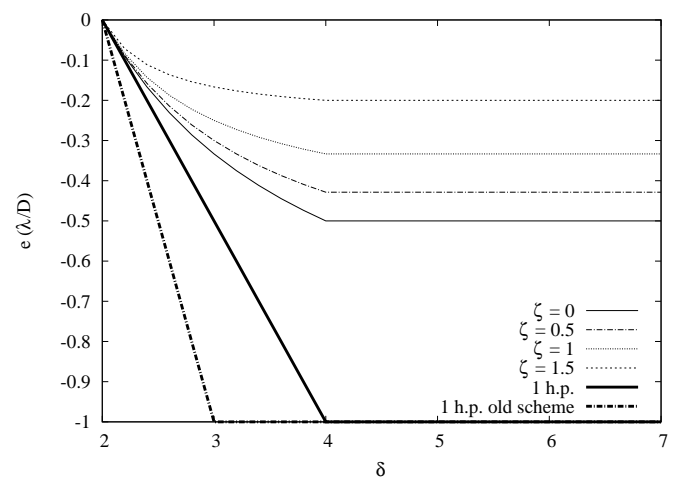

Figure 5: Scaling exponent of the power achievable by the proposed schemes as a function of $\delta$, for different values of $\zeta<2$ (multiple home-points) and for the single home-point case.

outperforms the one proposed in [22].

\subsection{Optimality of the proposed schemes}

In this section we show that our schemes jointly achieve nearoptimal throughput and delay performance, at least over a significant range of parameter values.

We start recalling two results that have been proved in [23].

Lemma 2. In a scenario in which nodes move around a single home-point, no scheduling-routing scheme can achieve a throughput larger than (in order sense):

$$
\lambda= \begin{cases}\Theta(1) & \delta \leq 2 \\ \Theta\left(n^{\frac{2-\delta}{2}(\log n)^{\frac{1-\delta}{2}}}\right) & 2 \leq \delta<3 \\ \Theta\left(n^{1 / 2}\right) & \delta \geq 3\end{cases}
$$

Lemma 3. In a scenario in which nodes move around two home-points, under the assumption $(\zeta<\delta)$ no scheduling-routing 
scheme can achieve a throughput that is in order sense larger than:

$$
\lambda= \begin{cases}\Theta(1 / \log n) & \zeta<2 \\ \Theta\left(n^{\frac{2-\zeta}{2}}\right) & 2 \leq \zeta<3 \\ \Theta\left(n^{1 / 2}\right) & \zeta \geq 3\end{cases}
$$

Comparing the results obtained by our schemes with the above upper bounds on the throughput, and observing that by construction $D=\Omega(1)$ under any possible scheme, we obtain:

Theorem 1. The proposed schemes jointly achieve optimal throughput and delay exponents for $2<\delta \leq 3$, in the case of single home-point, and for $2<\zeta \leq 3$, in the case of multiple home-points.

\section{CONCLUSIONS}

Previous work on asymptotic delay-throughput performance in mobile ad hoc networks has mostly been done under the assumption that the stationary distribution of each node is uniform over the network area. In [22] we have shown that this condition can be largely suboptimal. Indeed delay-throughput performance can be dramatically improved when each node moves around one home-point randomly placed in the area, according to a restricted mobility process which results into a spatial stationary distribution that decays as a power law of exponent $\delta$ with the distance from the home-point.

In this paper we have introduced a new class of scheduling and routing schemes that significantly outperform the schemes in [22] for $\delta>2$. In particular, the schemes introduced in this paper jointly achieve optimal throughput and delay scaling exponents for $2<\delta \leq 3$. We have also extended our analysis to the case of multiple home-points per node, showing that the network performance can be further improved by home-point multiplicity. In this case the proposed scheduling-routing schemes jointly achieve optimal throughput and delay scaling exponents for $2<\zeta \leq 3$.

\section{REFERENCES}

[1] P. Gupta, P.R. Kumar, "The capacity of wireless networks", IEEE Trans. on Information Theory, 46(2), pp. 388-404, 2000.

[2] M. Grossglauser, D.N.C. Tse, "Mobility increases the capacity of ad hoc wireless networks", IEEE/ACM Trans. on Networking, vol. 10, no. 2., August 2002.

[3] Delay Tolerant Network Research Group: www.dtnrg.org

[4] A. Chaintreau, P. Hui, J. Scott, R. Gass, J. Crowcroft, and C. Diot, "Impact of human mobility on opportunistic forwarding algorithms," IEEE Transactions on Mobile Computing, 6(6): 606-620, June 2007.

[5] J. Burgess, B. Gallagher, D. Jensen, B. N. Levine, "MaxProp: Routing for Vehicle-Based Disruption-Tolerant Networking", in Proc. IEEE INFOCOM, Barcelona, Spain, April 2006

[6] M. J. Neely, E. Modiano "Capacity and delay trade offs for ad-hoc mobile networks" IEEE Trans. on Information Theory, 51(6), pp. 1917-1937, 2005.

[7] L. Ying, S. Yang, R. Srikant, "Optimal Delay-Throughput Tradeoffs in Mobile Ad Hoc Networks," IEEE Trans. on Information Theory, 54(9), pp. 4119-4143, 2008.

[8] A. El Gamal, J. Mammen, B. Prabhakar, and D. Shah, "Throughput-Delay Trade-off in Wireless Networks", in Proc. IEEE INFOCOM '04.
[9] N. Bansal, Z. Liu, "Capacity, Delay and Mobility in Wireless Ad-Hoc Networks," in Proc. IEEE INFOCOM '03.

[10] G. Sharma, R. R. Mazumdar and N. B. Shroff, "Delay and Capacity Trade-offs in Mobile Ad Hoc Networks: A Global Perspective" in Proc. IEEE INFOCOM '06.

[11] J. H. Kang, W. Welbourne, B. Stewart, G. Borriello, "Extracting Places from Traces of Locations", ACM Mobile Computing and Communications Review, 9(3), July 2005.

[12] M. Balazinska, P. Castro, "Characterizing Mobility and Network Usage in a Corporate Wireless Local-Area Network," in Proc. MobiSys '03.

[13] N. Sarafijanovic-Djukic, M. Piorkowski, and M. Grossglauser, "Island Hopping: Efficient Mobility-Assisted Forwarding in Partitioned Networks", IEEE SECON 2006.

[14] J. Leguay, T. Friedman, V. Conan, "Evaluating Mobility Pattern Space Routing for DTNs", in Proc. IEEE INFOCOM '06.

[15] I. Rhee, M. Shin, K. Lee, S. Chong. S. Hong, "On the Levy-walk Nature of Human Mobility," in Proc. INFOCOM '08

[16] S.N. Diggavi, M. Grossglauser, D.N.C. Tse, "Even one-dimensional mobility increases ad hoc wireless capacity", IEEE Trans. on Information Theory, 51(11), pp. 3947-3954, 2005.

[17] J. Mammen and D. Shah, "Throughput and Delay in Random Wireless Networks with Restricted Mobility," IEEE Trans. on Information Theory, 53(3), pp. 1108-1116, 2007.

[18] M. Garetto, P. Giaccone, E. Leonardi, "Capacity Scaling in Delay Tolerant Networks with Heterogeneous Mobile Nodes" in Proc. ACM MobiHoc '07.

[19] M. Garetto, P. Giaccone, E. Leonardi, "Capacity Scaling of Sparse Mobile Ad Hoc Networks", in Proc. INFOCOM '08.

[20] F. Xue, P. R. Kumar, "Scaling laws for ad hoc wireless networks: an information theoretic approach", Found. Trends Netw., vol. 1, no. 2, pp. 145-270, 2006.

[21] L. Kleinrock, "On Flow Control in Computer Networks", in Proc. ICC '78.

[22] M. Garetto and E. Leonardi, "Restricted Mobility Improves Delay-Throughput Trade-offs in Mobile Ad-Hoc Networks," submitted for publication, available at http://arxiv.org/abs/0807.1228

[23] M. Garetto, P. Giaccone, E. Leonardi, "Capacity Scaling in Ad Hoc Networks with Heterogeneous Mobile Nodes: the Sub-critical Regime", accepted for publication on ACM/IEE Transactions on Networking available on-line at: http://www.telematica.polito.it/leonardi/papers/TON_sub.pdf

[24] H. Takagi, Queueing Analysis : Discrete-Time Systems, North Holland, 1993.

\section{APPENDIX}

\section{A. PROOF OF LEMMA 1}

Consider one node $a$ having a home point $H_{a}^{h}(h=1$ or 2$)$, within a cell $\mathcal{A}_{i}$ of area $A_{i}=\Omega(\log n)$. The probability that node $a$ is found in $\mathcal{A}_{i}$ can be evaluated as

$$
\mathbb{P}\left(a \in \mathcal{A}_{i} \mid H_{a}^{h} \in \mathcal{A}_{i}\right)>p_{H_{a}^{h}} \int_{\mathcal{A}_{i}} \phi\left(\| X-H_{a}^{h}||\right) \mathrm{d} X=\Theta(1)
$$

being $p_{H_{a}^{h}}=\Theta(1)$ the probability that node $a$ is associated to its $h$-th home-point. The integral in the above expression is $\Theta(1)$ for any $\delta>2$. Now, let $N_{H \in \mathcal{A}_{i}}$ be the number of nodes having one home-point in $\mathcal{A}_{i}$. The average number of nodes having one home- 
point in $\mathcal{A}_{i}$, that can be found in $\mathcal{A}_{i}$ is, by definition:

$$
\mathbb{E}\left[N_{\mathcal{A}_{i}}\right]=N_{H \in \mathcal{A}_{i}} \mathbb{P}\left(a \in \mathcal{A}_{i} \mid H_{a}^{h} \in \mathcal{A}_{i}\right)>\Theta\left(N_{H \in \mathcal{A}_{i}}\right)
$$

Since the home-points are Poisson distributed, whenever $A_{i}=$ $\Omega(\log n)$, the number of nodes $N_{H \in \mathcal{A}_{i}}$ having a home-point in $\mathcal{A}_{i}$ is w.h.p. $\Theta\left(A_{i}\right)$, and we get the assert.

\section{B. DIMENSIONING OF $\mathcal{A}_{i}$ FOR $i<i_{\max }$}

From Lemma 1 it directly descends that the average density of transmitters within $\mathcal{A}_{i}$ is $\Theta(1)$ w.h.p. whenever $A_{i}=\Omega(\log n)$, as it is given by $\mathbb{E}\left[N_{\mathcal{A}_{i}}\right] / A_{i}$.

Now we turn our attention to receivers $b$ and without lack of generality, we assume $\sqrt{2 A_{i}}<\epsilon z_{i}$ for some $\epsilon<<1$.

We remind that there are essentially three classes of potential $(i+1)$-relays $b$ that can be found in $\mathcal{A}_{i}$ : i) nodes $b$ having one home-point placed in the same $(i+1)$-level squarelet of the destination, and currently staying in $\mathcal{A}_{i}$ for effect of mobility around such home-point, at distance $\Theta\left(z_{i}\right)$. ii) nodes $b$ having one homepoint $H_{b}^{h}$ in the same $(i+1)$-level squarelet of the destination, another home-point $H_{b}^{3-h}$ placed in $\mathcal{A}_{i}$, and currently moving within $\mathcal{A}_{i}$ for effect of mobility around $H_{b}^{3-h}$. iii) nodes $b$ having one home-point $H_{b}^{h}$ in the same $(i+1)$-level squarelet of the destination, another home-point $H_{b}^{3-h}$ not placed in $\mathcal{A}_{i}$, and currently moving within $\mathcal{A}_{i}$ for effect of mobility around $H_{b}^{3-h}$.

Let us consider a node moving around a home-point placed in the same squarelet $S_{i+1}^{k}$ of the destination; the probability that this node is found in $\mathcal{A}_{i}$ is;

$$
\begin{aligned}
\mathbb{P}\left(b \in \mathcal{A}_{i} \mid H_{b}^{h} \in S_{i+1}^{k}\right) & =p_{H_{b}^{h}} \int_{\mathcal{A}_{i}} \phi\left(\left\|X-H_{b}^{h}\right\|\right) \mathrm{d} X \\
& =\Theta\left(A_{i} z_{i}^{-} \delta\right)
\end{aligned}
$$

Thus, considering that the number of nodes having a home-point in $S_{i+1}^{k}$ is $\Theta\left(z_{i}^{2}\right)$, the average density of such nodes in $\mathcal{A}_{i}$ is $\Phi_{i}^{1}=$ $z_{i}^{2-\delta}$.

Now we evaluate the probability that a node $b$ having one homepoint $H_{b}^{h}$ (with $h=1$ or 2 ) in $S_{i+1}^{k}$ has the other home point $H_{b}^{3-h}$ in a cell $\mathcal{A}_{i}$ located at distance $z_{i}$ from $S_{i+1}^{k}$ :

$$
\begin{aligned}
\mathbb{P}\left(H_{b}^{3-h} \in \mathcal{A}_{i} \mid H_{b}^{h} \in S_{i+1}^{k}\right) & =\int_{\mathcal{A}_{i}} \psi\left(\left\|X-H_{b}^{h}\right\|\right) \mathrm{d} X \\
& =\Theta\left(\frac{A_{i} z_{i}^{-\zeta}}{G(\zeta)}\right)
\end{aligned}
$$

Now, considering that the number of nodes having one home-point in $S_{i+1}^{k}$ is $\Theta\left(z_{i}^{2}\right)$ and that nodes having an home-point in $\mathcal{A}_{i}$ have a non-null probability to be found in $\mathcal{A}_{i}$, we easily get that $\Phi_{i}^{2}=$ $\frac{z_{i}^{2-\zeta}}{G(\zeta)}$

At last we evaluate the probability that a node $b$ having one homepoint $H_{b}^{h}$ (with $h=1$ or 2 ) in $S_{i+1}^{k}$, and the other home-point $H_{b}^{3-h}$ outside $\mathcal{A}_{i}$, can be found in $\mathcal{A}_{i}$.

$$
\begin{aligned}
& \mathbb{P}\left(b \in \mathcal{A}_{i}, H_{a}^{3-h} \notin \mathcal{A}_{i} \mid H_{b}^{h} \in S_{i+1}^{k}\right)= \\
& \quad=p_{H_{b}^{3-h}} \int_{\mathcal{A}_{i}} \int_{\mathcal{O} \backslash \mathcal{A}_{i}} \phi\left(\left\|X-H_{b}^{3-h}\right\|\right) \psi\left(\left\|H_{b}^{3-h}-H_{b}^{h}\right\|\right) \\
& \mathrm{d} H_{b}^{3-h} \mathrm{~d} X
\end{aligned}
$$

Defining as $\mathcal{B}_{X, \epsilon}$ the $\mathbb{R}^{2}$-ball of center $X$ and radius $\epsilon z_{i}$, we split the inner integral into the sum of three contributions. The first is computed over an area $\mathcal{B}_{H_{b}^{h}, \epsilon}$ : in this case the term $\psi\left(\| H_{b}^{3-h}-\right.$
$\left.H_{b}^{h} \|\right)$ can be considered $\Theta(1)$. Taking into account the triangular inequality $\left\|X-H_{b}^{3-h}\right\|+\left\|H_{b}^{3-h}-H_{b}^{h}\right\| \geq\left\|X-H_{b}^{h}\right\|=\Theta\left(z_{i}\right)$, the first contribution becomes:

$$
\begin{aligned}
& \int_{\mathcal{A}_{i}} \int_{\mathcal{B}_{H_{b}^{h}, \epsilon}} \phi\left(\left\|X-H_{b}^{3-h}\right\|\right) \mathrm{d} H_{b}^{3-h} \mathrm{~d} X \\
& \leq \int_{\mathcal{A}_{i}} \int_{\mathcal{B}_{H_{b}^{h}}, \epsilon} \phi\left(\left\|X-H_{b}^{h}\right\|\right) \mathrm{d} H_{b}^{3-h} \mathrm{~d} X \\
& \quad=O\left(\Phi_{i}^{1}\right)
\end{aligned}
$$

The second contribution is computed on $\mathcal{B}_{X, \epsilon} \backslash \mathcal{A}_{i}$ : using the same considerations above, we can say that it is $O\left(\Phi_{i}^{2}\right)$. At last, the third contribution computed over $\mathcal{O} \backslash\left(\mathcal{B}_{H_{b}, \epsilon} \cup \mathcal{B}_{X, \epsilon}\right)$ can be easily shown to be negligible. Indeed, note that uniformly over the considered domain we have: $\frac{\epsilon}{1+\epsilon} \leq \frac{|| X-H_{b}^{3-h} \mid}{\left\|H_{b}^{3-h}-H_{b}^{h}\right\|} \leq \frac{1+\epsilon}{\epsilon}$.

Thus, being function $\psi()$ non increasing we have:

$$
\begin{gathered}
\int_{\mathcal{A}_{i}} \int_{\mathcal{O} \backslash\left(B_{H_{b}, \epsilon} \cup \mathcal{B}_{X, \epsilon}\right)} \phi\left(\left\|X-H_{b}^{3-h}\right\|\right) \\
\psi\left(\left\|H_{b}^{3-h}-H_{b}^{h}\right\|\right) \mathrm{d} H_{b}^{3-h} \mathrm{~d} X \\
\leq \int_{\mathcal{A}_{i}} \int_{\mathcal{O} \backslash\left(B_{H_{b}, \epsilon} \cup \mathcal{B}_{X, \epsilon}\right)} \phi\left(\left\|X-H_{b}^{3-h}\right\|\right) \\
\psi\left(\frac{\epsilon}{1+\epsilon}\left\|X-H_{b}^{3-h}\right\|\right) \mathrm{d} H_{b}^{3-h} \mathrm{~d} X \\
=\Theta\left(A_{i} z_{i}^{2-(\delta+\zeta)}\right)
\end{gathered}
$$

Combining previous results we can conclude that $\Phi_{i}^{3}=$ $O\left(\max \left(\Phi_{i}^{1}, \Phi_{i}^{2}\right)\right)$

\section{EVALUATION OF THE NUMBER OF NODES AT DISTANCE $\Theta\left(z_{i}\right)$ FROM THE HOME-POINT}

For the sake of the brevity we restrict to the case of a single home point. The generalization to the multiple home-point case can be easily carried out following the same rationale as in Appendix B.

Let us consider a node $a$ having a home-point in $\mathcal{A}_{i}$. The probability that this node is found at a distance $\Theta\left(z_{i}\right)$ from its home-point is:

$$
\mathbb{P}\left(z_{i}\right)=2 \pi \int_{c z_{i}}^{C z_{i}} \rho \phi(\rho) \mathrm{d} \rho=\Theta\left(z_{i}^{2-\delta}\right)
$$

being $0<c<C<\infty$ two arbitrary constants. Now, since the number $N_{H \in \mathcal{A}_{i}}$ of nodes having one home-point in $\mathcal{A}_{i}$ is, w.h.p, $\Theta\left(A_{i}\right)$ and being $A_{i}=z_{i}^{\delta-2}$, it follows that the average number of nodes having one home point in $\mathcal{A}_{i}$ that are found at distance $\Theta\left(z_{i}\right)$ from their home-point is $\mathbb{P}\left(z_{i}\right) N_{H \in \mathcal{A}_{i}}=\Theta(1)$. 